\title{
Early bearing and vegetative growth of 153 Sicilian olive accessions
}

Placido Volo, Silvia Fretto, Riccardo Lo Biancoํㅡ, Laura Macaluso, Tiziano Caruso

Department of Agricultural, Food and Forest Sciences, University of Palermo, Viale delle Scienze, 90128 Palermo, Italy

\section{Abstract}

In 2014, an olive germplasm collection was established in southwestern Sicily, Italy. The collection included 153 Sicilian accessions and 3 international varieties (reference), each with 7 trees, spaced at $5 \times 2 \mathrm{~m}$ and trained to hedgerow system. The aim of the trial was to study early growth and yield responses of the accessions to superhigh planting density (SHD). Trunk cross sectional area, tree height, pruning weights, shoot positioning, blooming and fruit yield (kg/tree) were measured in 2015 and 2016. Based on trunk growth and amount of prunings, the 153 accessions were separated into four categories of vigor as it follows: very high vigor (6 accesions ), high vigor (13), medium vigor (131), and low vigor (3). In $85 \%$ of the accessions, the majority of shoots were born on the central part of the main stem, indicating prevalent mesotony. Constant early blooming (100\% of trees in trial in both years) was recorded in 7 accessions ('Nebba 1', 'Nocellara di Licata', 'Olivo di Monaci', 'Olivo di Monaci 1', 'Olivo di Mandanici', 'Verdella', and 'Calatina'), with 'Nocellara di Licata' and 'Verdella' bearing no fruit at the end. Accessions in the very high and low vigor categories produced no fruit, determining a lack of relationship between early bearing and tree vigor.

Keywords: blooming, hedgerow, Olea europaea, super high density plantings, tree vigor, trunk growth

\section{INTRODUCTION}

In Sicily, over $65 \%$ of olive trees are grown in rainfed traditional plantings using vigorous trees trained to open canopies and planted at wide spacings (low density plantings). Those groves are characterized by relatively late productions, low fruit and oil yields, biennial bearing and high management costs (mostly hand labor), often generating very low or no income. On the other hand, olive oils from Sicilian cultivars are characterized by unique sensory attributes and generally high quality standards (Galvano et al., 2007).

On the contrary, modern super high density (SHD) plantings are generally characterized by early, constant and high yields per hectare and

1 Email: riccardo.lobianco@unipa.it 
can be mechanically harvested (Tous et al., 2005; De Gennaro, 2012; Connor et al., 2014). They are established using low vigor trees at reduced spacings (over 1500 trees/ha) (Del Rio et al., 2002), and the 'Arbequina' olive has proven to be the most suitable (and by far most widely used) cultivar for those planting systems (Connor et al., 2014). Adequate light interception by hedgerows is critical for good and constant bearing as much as for high quality productions. In SDH plantings, the latter can be accomplished by increasing planting densities and training trees to a central leader (Tous et al., 2005). To date, only a very limited number of cultivars have shown regular yields and adequate vigor adapting well to SDH planting systems.

A selection program has been started recently at the Department of Agricultural, Food and Forest Sciences of the University of Palermo to collect and screen several Sicilian olive accessions for vegetative and reproductive traits suitable for hedgerow high density plantings. Ultimately, the aim of the program is to find genotypes that combine together early and constant bearing, growth and fruiting habits suitable for hedgerow high density plantings along with the distinctive olive oil quality typical of the major Sicilian cultivars. For this purpose, experimental plantings have been established to form hedgerows with optimum light interception throughout the canopy (from top to base) to be tested for fruiting efficiency and early/regular bearing.

\section{MATERIALS AND METHODS}

The study was conducted in 2014-2016 in an experimental plot located in southwestern Sicily ( $37^{\circ} 35^{\prime} \mathrm{N}, 12^{\circ} 54^{\prime} \mathrm{E}, 58 \mathrm{~m}$ a.s.l.) using one-year-old trees of 153 different local olive accessions (Table 1) grafted on 'Nocellara del Belice' clonal rootstock. In fall 2014, seven trees of each accession were planted consecutively at spacings of $5 \times 2 \mathrm{~m}$ (1000 trees/ha). The plot was managed using proper cultivation practices, including mechanical weed control and irrigation (about $60 \mathrm{~mm} /$ year) during summer months. Trees were left unpruned while in the nursery and during the first year in the field. Starting in February 2015, hedgerows were formed by removing inter-roworiented shoots/branches. Growth was estimated by measuring number of shoots and their position along the main stem, tree height, trunk circumference (10 $\mathrm{cm}$ above the grafting point) and by recording pruned biomass. Trunk circumference was used to calculate trunk cross-section area (TCSA) and estimate growth rate across years.

Percentage of blooming trees was recorded in 2015 and 2016; early yields were recorded in 2016. Growth data were analyzed using graphical statistics (analysis of means) and the accessions were separated into four main vigor categories based on pruned biomass and trunk growth rate (AGR).

\section{RESULTS AND DISCUSSION}


Field and nursery measurements of plant size and pruned biomass indicated high growth variability and a greater number of Sicilian accessions showing above-average vigor. Considering pruned biomass and trunk growth together, the 153 accessions were separated into four categories of vigor as it follows: very high vigor (6), high vigor (13), medium vigor (131), and low vigor (3). Specifically, BA1, BS, CE4, SPA, VCA, and VLA exhibited very high vigor, while only IM exhibited very low vigor (Figure 1). Similar tendencies were confirmed by shoot positioning along the main stem (Table 1). In particular, the vast majority of the accessions had most of the shoots in the central portion of the main stem (prevalent mesotony), 25 accessions exhibited some tendency toward acrotony, while only six accessions exhibited some tendency toward basitony.

Seven accessions (CAL, NEB1, NOL, ODM, ODM5, OMD, VLA) showed early and constant blooming whereas only 19 accessions developed a detectable amount of fruit in 2016 (Table 1). Absolutely no significant relationship was observed between vegetative growth (AGR, pruning weights or TCSA) and fruit yield (data not shown), indicating that there is good potential for individuating efficient genotypes suitable for high density plantings, with low vigor and good yields. Future years of investigation are needed to confirm these preliminary trends.

\section{Literature cited}

Tous, J., Romero, A., Plana, J. and Hermoso, J.F. (2005). Ensayo de densidades altas de plantación en la variedad de olivo 'Arbequina'. In: V Congreso Ibérico de Ciencias Hortícolas. Porto, Portugal, May (pp. 2227).

Connor, D.J., Gómez-del-Campo, M., Rousseaux, M.C. and Searles, P.S. (2014). Structure, management and productivity of hedgerow olive orchards: a review. Sci. Hortic. 169, 71-93.

Del Rio, C., Garcia-Fernandez M.D. and Caballero J.M. (2002). Variability and classification of olive cultivars by their vigor. Acta Hortic. 586, 229-232.

De Gennaro, B., Notarnicola, B., Roselli, L. and Tassielli, G. (2012). Innovative olive growing models: an environmental and economic assessment. J. Cleaner Prod. 28, 70-80.

Galvano, F., La Fauci, L., Graziani, G., Ferracane, R., Masella, R., Di Giacomo, C., Sacco, A., D’Archivio, M., Vanella, L. and Galvano, G. (2007). Phenolic compounds and antioxidant activity of Italian extra virgin olive oil Monti Iblei. J. Medicinal Food 10, 650-656. 
Table 1. Names and abbreviations of the Sicilian olive accessions in trial, percent distribution of one-year-old shoots along the main stem in the nursery (2014), presence/absence (YES/NO) of blooming in the field (YES = $100 \%$ of blooming trees) and yield ( $\mathrm{kg} /$ tree).

\begin{tabular}{|c|c|c|c|c|c|c|c|}
\hline \multirow[b]{2}{*}{ Accession name } & \multirow[b]{2}{*}{ Abbreviation } & \multicolumn{3}{|c|}{ Shoot position (\%) } & \multicolumn{2}{|c|}{ Blooming } & \multirow{2}{*}{$\begin{array}{l}\text { Yield } \\
2016\end{array}$} \\
\hline & & Basal & Central & Apical & 2015 & 2016 & \\
\hline Abunara & $A B$ & 10 & 51 & 39 & No & No & - \\
\hline Aitana & AT & 24 & 54 & 22 & No & No & - \\
\hline Bariddara 1 & BA1 & 21 & 68 & 11 & No & No & - \\
\hline Bariddara 2 & BA2 & 32 & 28 & 39 & No & No & - \\
\hline Biancolilla caltabellotta & $\mathrm{BC}$ & 9 & 65 & 26 & No & Yes & 2.84 \\
\hline Biancolilla caltabellotta PC & $\mathrm{BCC}$ & 15 & 43 & 42 & No & Yes & 1.82 \\
\hline Biancolilla Di Marco & BDM & 12 & 67 & 21 & No & Yes & 0 \\
\hline Bottone di gallo & BG & 14 & 60 & 26 & No & No & - \\
\hline Bottone di gallo 1 & BG1 & 19 & 58 & 23 & No & Yes & 1.23 \\
\hline Biancolilla iacapa & $\mathrm{BI}$ & 4 & 78 & 18 & No & No & - \\
\hline Biancolilla misilmeri & BM & 0 & 53 & 47 & No & Yes & 0 \\
\hline Biancolilla napoletana & BN & 14 & 52 & 34 & No & No & - \\
\hline Biancolilla pantelleria & $\mathrm{BP}$ & 6 & 58 & 36 & No & Yes & 0 \\
\hline Brandofino & $\mathrm{BR}$ & 12 & 44 & 44 & No & Yes & 0 \\
\hline Brandofino 1 & BR1 & 11 & 30 & 60 & No & Yes & 0 \\
\hline Biancolilla siracusana & BS & 29 & 55 & 16 & No & No & - \\
\hline Biancolilla Schimmenti & BSC & 10 & 63 & 27 & No & Yes & 2.54 \\
\hline Bella di Spagna & BSP & 13 & 54 & 33 & No & Yes & 0 \\
\hline Biancolilla tiziano & $\mathrm{BT}$ & 6 & 75 & 19 & No & No & - \\
\hline Biancuzza & $B Z$ & 15 & 38 & 47 & No & Yes & 0 \\
\hline Cacaredda & $\mathrm{CA}$ & 50 & 41 & 9 & No & Yes & 0 \\
\hline Cacaredda 1 & CA1 & 28 & 51 & 21 & No & No & - \\
\hline Cacazzara & CAC & 17 & 48 & 35 & No & No & - \\
\hline Cacaridduni 1 & CAD1 & 15 & 69 & 16 & No & No & - \\
\hline Cacaridduni 2 & CAD2 & 7 & 76 & 17 & No & No & - \\
\hline Calatina & CAL & 15 & 52 & 33 & Yes & Yes & 1.77 \\
\hline Calamignara & CAM & 25 & 40 & 35 & No & No & - \\
\hline Castricianella Rapparina & CAR & 20 & 55 & 25 & No & Yes & 1.94 \\
\hline Castelluzzo & CAS & 30 & 40 & 30 & No & No & - \\
\hline Cavalieri & CAV & 18 & 57 & 24 & No & Yes & 0 \\
\hline Cacazzana & CAZ & 20 & 52 & 28 & No & No & - \\
\hline Cerasuola Cappuccia & $\mathrm{CC}$ & 11 & 62 & 27 & No & Yes & 0 \\
\hline Cerasuola & CE & 1 & 77 & 22 & No & No & - \\
\hline Cerasuola 1 & CE1 & 2 & 65 & 32 & No & Yes & 0 \\
\hline Cerasuola 2 & CE2 & 8 & 70 & 22 & No & No & - \\
\hline Cerasuola 3 & CE3 & 26 & 60 & 14 & No & Yes & 0 \\
\hline Cerasuola 4 & CE4 & 19 & 62 & 19 & No & No & - \\
\hline Citrale & CIT & 7 & 73 & 20 & No & No & - \\
\hline Conservolia & CON & 14 & 45 & 41 & No & No & - \\
\hline Crastu & $\mathrm{CR}$ & 23 & 42 & 35 & No & Yes & 2.10 \\
\hline Crastu 1 & CR1 & 21 & 33 & 47 & No & No & - \\
\hline Erbano & ER & 34 & 41 & 25 & No & Yes & 1.58 \\
\hline Fastucara aragona & FA & 4 & 48 & 48 & No & No & - \\
\hline Galatina & GA & 5 & 65 & 29 & No & No & - \\
\hline Giarraffa & GF & 20 & 45 & 34 & No & No & - \\
\hline
\end{tabular}




\begin{tabular}{|c|c|c|c|c|c|c|c|}
\hline Giarraffa 1 & GF1 & 48 & 35 & 17 & No & No & - \\
\hline Giarraffa 2 & GF2 & 18 & 39 & 42 & No & No & - \\
\hline Giarraffa 3 & GF3 & 20 & 37 & 43 & No & No & - \\
\hline Giarraffa 4 & GF4 & 16 & 66 & 18 & No & No & - \\
\hline Ghiannuzza & $\mathrm{GH}$ & 0 & 79 & 21 & No & No & - \\
\hline Gordales & GO & 16 & 48 & 36 & No & Yes & 0 \\
\hline lacona & $\mathrm{IA}$ & 8 & 54 & 37 & No & No & - \\
\hline Impressionante & IM & 9 & 83 & 9 & No & No & - \\
\hline Indemoniata & IND & 5 & 79 & 17 & No & No & - \\
\hline Leucocarpa & LE & 6 & 52 & 42 & No & No & - \\
\hline Leucocarpa 1 & LE1 & 11 & 39 & 50 & No & No & - \\
\hline Lumiaru & LU & 2 & 56 & 42 & No & Yes & 0 \\
\hline Lunga di Vassallo & LUV & 28 & 33 & 39 & No & Yes & 0 \\
\hline Marfia & MAF & 17 & 63 & 20 & No & No & - \\
\hline Marsalesa & MAR & 10 & 68 & 22 & No & No & - \\
\hline Minna di vacca & MDV & 8 & 76 & 16 & No & Yes & 0 \\
\hline Minuta & MN & 11 & 67 & 22 & No & Yes & 1.71 \\
\hline Minuta 1 & MN1 & 22 & 56 & 22 & No & Yes & 1.31 \\
\hline Monaca & MO & 19 & 44 & 37 & No & Yes & 0 \\
\hline Montonica & MON & 16 & 62 & 22 & No & No & - \\
\hline Mortillara & MOR & 37 & 37 & 26 & No & No & - \\
\hline Moresca & MR & 3 & 67 & 30 & No & No & - \\
\hline Moresca 1 & MR1 & 4 & 51 & 44 & No & No & - \\
\hline Moresca 2 & MR2 & 1 & 60 & 38 & No & No & - \\
\hline Moresca 3 & MR3 & 0 & 71 & 29 & No & No & - \\
\hline Moresca 4 & MR4 & 0 & 69 & 31 & No & No & - \\
\hline Moresca di noto & MRN & 0 & 50 & 50 & No & Yes & 0 \\
\hline Murtiddara & MU & 10 & 58 & 32 & No & No & - \\
\hline Murtiddara 1 & MU1 & 7 & 67 & 25 & No & No & - \\
\hline Nasitana & NA & 24 & 40 & 36 & No & Yes & 0.98 \\
\hline Nebba & NEB & 29 & 38 & 33 & No & Yes & 1,18 \\
\hline Nebba 1 & NEB1 & 19 & 54 & 27 & Yes & Yes & 1.67 \\
\hline Nerba & NER & 10 & 58 & 32 & No & No & - \\
\hline Nerba 1 & NER1 & 11 & 47 & 41 & No & Yes & 0 \\
\hline Nerba sangue dolce & NESD & 14 & 71 & 16 & No & Yes & 0 \\
\hline Nocellara del Belice & NOB & 10 & 70 & 20 & No & No & - \\
\hline Nocellara del Belice 1 & NOB1 & 18 & 72 & 10 & No & Yes & 0 \\
\hline Nocellara del Belice 2 & NOB2 & 22 & 68 & 10 & No & No & - \\
\hline Nocellara del Belice 3 & NOB3 & 23 & 71 & 6 & No & No & - \\
\hline Nocellara del belice 4 & NOB4 & 22 & 55 & 22 & Yes & No & - \\
\hline Nocellara del Belice 5 & NOB5 & 18 & 70 & 12 & No & Yes & 0 \\
\hline Nocellara del Belice 6 & NOB6 & 27 & 53 & 21 & No & Yes & 0 \\
\hline Nocellara del Belice 7 & NOB7 & 28 & 57 & 15 & No & No & - \\
\hline Nocellara Castelvetrano & NOC & 20 & 66 & 14 & No & No & - \\
\hline Nocellara Etnea & NOE & 59 & 16 & 26 & No & No & - \\
\hline Nocellara licata & NOL & 17 & 60 & 22 & Yes & Yes & 0 \\
\hline Nocellara messinese & NOM & 17 & 45 & 38 & No & Yes & 0 \\
\hline Nocellara messinese 2 & NOM2 & 2 & 57 & 42 & No & No & - \\
\hline Nocellara messinese 3 & NOM3 & 0 & 58 & 42 & No & Yes & 0 \\
\hline Nocellara messinese 4 & NOM4 & 0 & 86 & 14 & No & Yes & 2.53 \\
\hline Nocellara semenzale & NOS & 26 & 38 & 36 & No & Yes & 0 \\
\hline Nostrale Terminisa & NOT & 9 & 66 & 25 & No & No & - \\
\hline
\end{tabular}




\begin{tabular}{|c|c|c|c|c|c|c|c|}
\hline Olivo di Monaci & ODM & 21 & 45 & 34 & Yes & Yes & 2.08 \\
\hline Olivo di Monaci 1 & ODM1 & 24 & 40 & 37 & Yes & Yes & 2.27 \\
\hline Ogliara & OG & 8 & 57 & 35 & Yes & No & - \\
\hline Ogliara 1 & OG1 & 10 & 78 & 13 & No & No & - \\
\hline Ogliarola licata & OGL & 13 & 78 & 8 & No & No & - \\
\hline Ogliarola Nostrale & OGN & 12 & 65 & 23 & No & No & - \\
\hline Oliva Longa & $\mathrm{OL}$ & 20 & 61 & 19 & No & No & - \\
\hline Ogliarola Messinese & OM & 33 & 50 & 17 & No & No & - \\
\hline Ogliarola Messinese 1 & OM1 & 53 & 23 & 24 & No & No & - \\
\hline Ogliarola Messinese 2 & OM2 & 47 & 36 & 17 & No & No & - \\
\hline Olivo di Mandanici & OMD & 18 & 65 & 17 & Yes & Yes & 1.40 \\
\hline Opera Pia & $\mathrm{OP}$ & 15 & 74 & 10 & No & Yes & 0 \\
\hline Oliva saracena & OS & 0 & 71 & 29 & No & No & - \\
\hline Olivo di vicari & OV & 17 & 52 & 31 & No & No & - \\
\hline Pizzo di corvo & PC & 20 & 57 & 23 & No & No & - \\
\hline Picholine & PIC & 15 & 55 & 30 & Yes & No & - \\
\hline Pizzuta D'Olio & PID & 24 & 55 & 20 & No & No & - \\
\hline Pirunara & PIR & 36 & 51 & 13 & No & No & - \\
\hline Pirunara 1 & PIR1 & 29 & 52 & 19 & No & Yes & 0 \\
\hline Pizzutella 1 & PIZ1 & 18 & 63 & 19 & No & Yes & 1.16 \\
\hline Pizzutella 2 & PIZ2 & 11 & 47 & 42 & No & No & - \\
\hline Pizzutella 3 & PIZ3 & 11 & 89 & 0 & No & No & - \\
\hline Piricuddara & PRC & 23 & 50 & 26 & No & Yes & 1.16 \\
\hline Passulunara & PS & 9 & 64 & 27 & No & Yes & 0 \\
\hline Passulunara 1 & PS1 & 34 & 60 & 6 & No & No & - \\
\hline Passulunara 2 & PS2 & 20 & 46 & 34 & No & No & - \\
\hline Passulunara 3 & PS3 & 6 & 49 & 46 & No & No & - \\
\hline Passulunara 4 & PS4 & 15 & 39 & 46 & No & No & - \\
\hline Raitana & RAl & 4 & 79 & 17 & No & No & - \\
\hline Rizza Caccamo & RIC & 28 & 67 & 5 & No & No & - \\
\hline Rizzo & RIZ & 15 & 61 & 24 & No & No & - \\
\hline Rotondella & RO & 6 & 56 & 38 & No & No & - \\
\hline Santagatese & SA & 20 & 56 & 24 & No & No & - \\
\hline Sammartinara & SAM & 16 & 57 & 28 & No & No & - \\
\hline Scognita & $\mathrm{SC}$ & 5 & 88 & 8 & No & No & - \\
\hline Santa Caterina & SCT & 26 & 64 & 10 & No & No & - \\
\hline Spagnola Aragona & SPA & 15 & 59 & 26 & No & No & - \\
\hline Salicina vassallo & SV & 4 & 74 & 22 & No & Yes & 0 \\
\hline Tonda dolce & TD & 34 & 55 & 11 & No & Yes & 0 \\
\hline Tonda Iblea & $\mathrm{TI}$ & 14 & 44 & 42 & No & No & - \\
\hline Tortella Morticiana & TM & 9 & 77 & 14 & No & No & - \\
\hline Tunnulidda & TU & 20 & 51 & 29 & No & Yes & 0 \\
\hline Tunnulidda 1 & TU1 & 28 & 72 & 0 & No & No & - \\
\hline Uovo di piccione & UP & 20 & 62 & 19 & No & Yes & 0 \\
\hline Vaddarica & VCA & 9 & 55 & 36 & Yes & No & - \\
\hline Vaddarica 1 & VCA1 & 27 & 63 & 10 & No & Yes & 0 \\
\hline Vetrana & VET & 28 & 46 & 26 & No & Yes & 0 \\
\hline Verdella & VLA & 13 & 54 & 34 & Yes & Yes & 0 \\
\hline Verdella 1 & VLA1 & 9 & 65 & 26 & Yes & No & - \\
\hline Verdello & VLO & 22 & 39 & 39 & No & No & - \\
\hline Verdello 1 & VLO1 & 14 & 54 & 32 & No & No & - \\
\hline Vaddara & VRA & 21 & 55 & 24 & Yes & No & - \\
\hline
\end{tabular}




\begin{tabular}{llllllll} 
Verdese & VSE & 24 & 50 & 27 & No & No & - \\
Zaituna 1 & ZA1 & 25 & 49 & 26 & No & No & - \\
Zaituna 2 & ZA2 & 6 & 63 & 31 & No & No & - \\
Zaituna 3 & ZA3 & 10 & 59 & 31 & No & No & - \\
\hline
\end{tabular}




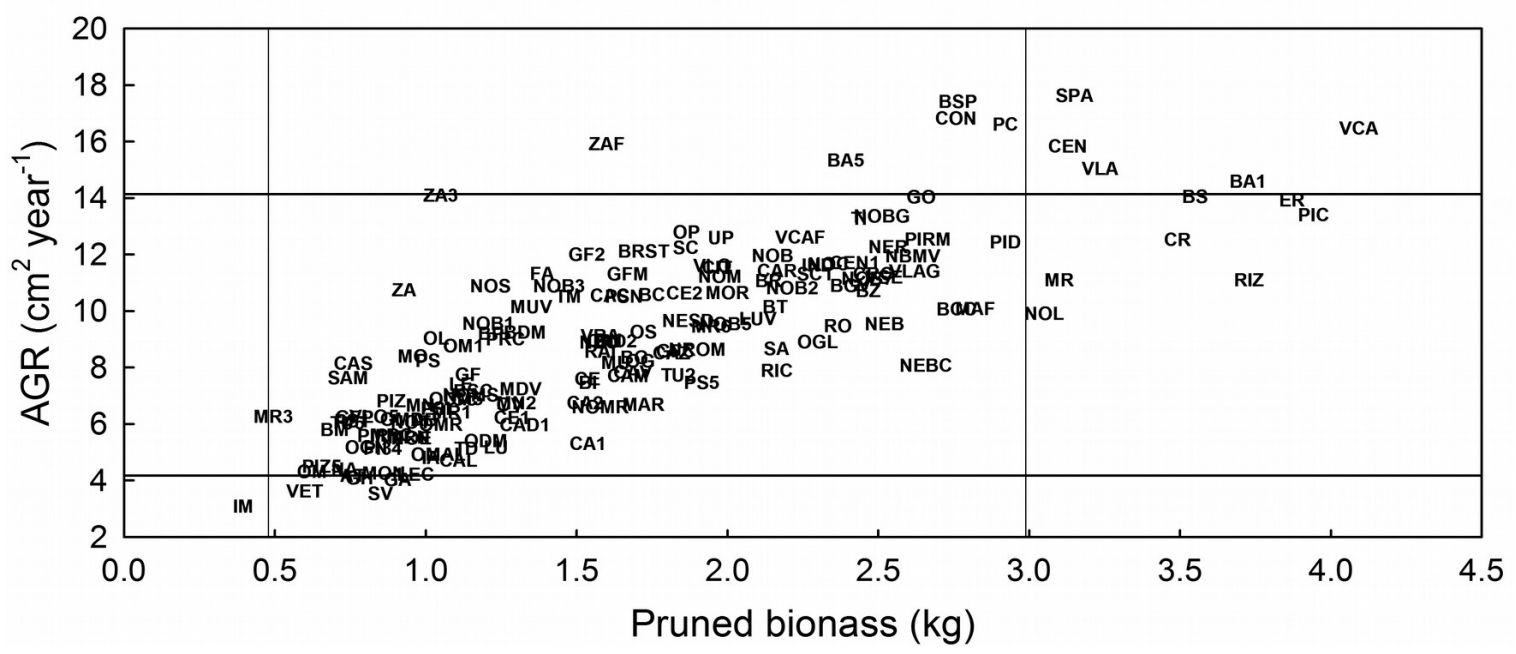

Figure 1. Association between pruned biomass (2016) and trunk growth rate (AGR) in the 153 olive accessions grown in the field. Vertical lines indicate upper and lower decision limits from analysis of means for pruned biomass, horizontal lines indicate upper and lower decision limits for AGR. 